\title{
Co-occurring symptoms in older oncology patients with distinct attentional function profiles
}

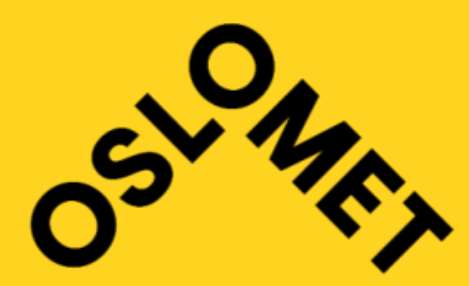

\author{
I. Utne ${ }^{1}$, B. Løyland ${ }^{1}$, E.K. Grov¹, S.M. Paul², B.A. Cooper², C. Miaskowski². \\ ${ }^{1}$ Oslo Metropolitan University - Faculty of Health Sciences, Department of Nursing and Health Promotion, Oslo, Norway. \\ 2University of California - San Francisco - Schools of Nursing, Department of Physiological Nursing, San Francisco, USA.
}

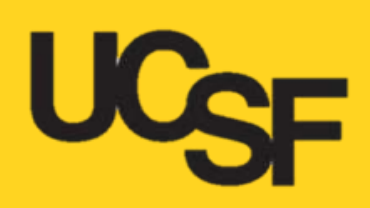

\section{BACKGROUND}

Attentional function (AFI) is an extremely important outcome for older oncology patients. In previous work, we identified three distinct attentional functional profiles (i.e., low (36.7\%), moderate (37.3\%), and high (26.0\%)).

The aims of this study were to:

- Evaluate how subgroups of patients with distinct AFI profiles differ on the severity of nine common symptoms, and

- Determine demographic and clinical characteristics and symptom severity scores associated with membership in the AFI classes.

\section{Distinct attentional function profiles}

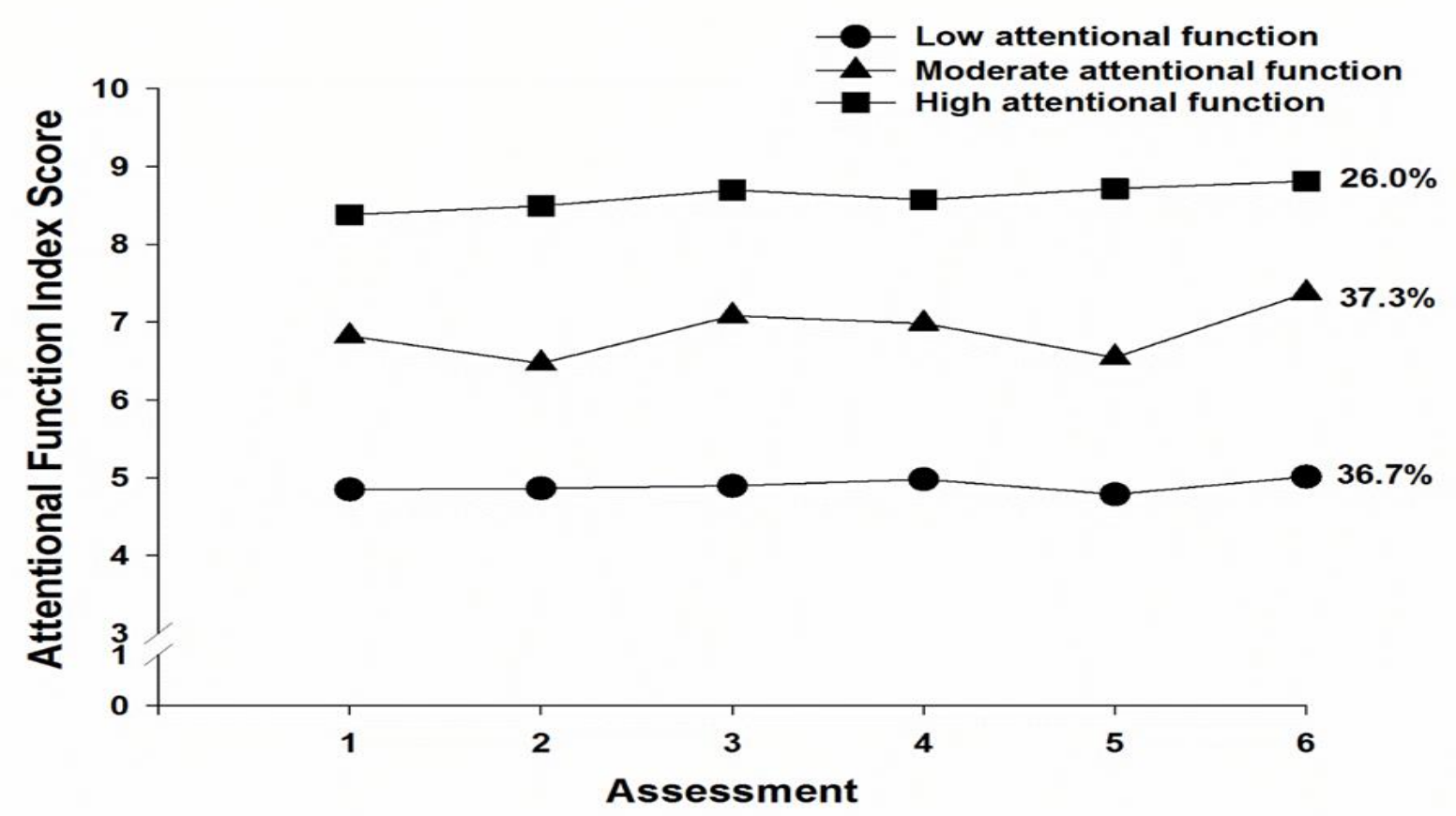

\section{METHODS}

\section{Inclusion criteria patients}

- Adults > 65 years old

- Breast, gastrointestinal, gynaecological, or lung cancer

- Received Chemotherapy (CTX) the preceding four weeks

- Scheduled to receive at least two additional cycles of CTX

\section{Participants}

- Patients $(n=365)$ with mean age $71.4(+5.5)$

\section{Statistics}

Descriptive statistics

Logistic regression analysis

\section{Instruments}

- Demographic questionnaire

- Attentional Function Index (AFI)

- Self-Administered Comorbidity Questionnaire (SCQ)

- Center for Epidemiological Studies-Depression Scale (CES-D)

- Spielberger State Anxiety Inventory (STAI-T)

- Spielberger Trait Anxiety Inventory (STAI-T)

- General Sleep Disturbance Scale (GSDS)

- Lee Fatigue Scale (LFS)

- Brief Pain Inventory (BPI)

\section{RESULTS}

For most of the symptoms, differences in severity scores followed the same pattern (i.e., low > moderate $>$ high class). No age, education, or gender differences.

For the regression analysis - Patients in the low AFI class were less likely to be married and employed, had lower annual household income, had higher level of comorbidity, reported more depression and back pain, and had higher CTX toxicity score.

\section{Co-Occurring Symptom Severity Scores}

\begin{tabular}{|l|c|c|c|c|}
\hline \multirow{2}{*}{ Symptoms } & $\begin{array}{c}\text { Low }(0) \\
36.7 \% \\
(\mathrm{n}=134)\end{array}$ & $\begin{array}{c}\text { Moderate }(1) \\
37.3 \% \\
(\mathrm{n}=136)\end{array}$ & $\begin{array}{c}\text { High }(2) \\
26.0 \% \\
(\mathrm{n}=95)\end{array}$ & \multirow{2}{*}{ Statistics } \\
\cline { 2 - 4 } Mean (SD) & Mean (SD) & Mean (SD) & \\
\hline Trait anxiety & $40.5(11.0)$ & $32.7(8.1)$ & $26.2(6.2)$ & $\begin{array}{c}\mathrm{F}=71.71, \mathrm{p}<.001 \\
\mathbf{0}>\mathbf{1}>\mathbf{2}\end{array}$ \\
\hline State anxiety & $38.9(13.2)$ & $30.8(9.9)$ & $24.7(6.9)$ & $\begin{array}{c}\mathrm{F}=50.15, \mathrm{p}<.001 \\
\mathbf{0}>\mathbf{1}>\mathbf{2}\end{array}$ \\
\hline Depressive & $16.8(10.0)$ & $9.9(7.3)$ & $4.6(4.0)$ & $\begin{array}{c}\mathrm{F}=69.89, \mathrm{p}<.001 \\
\mathbf{0}>\mathbf{1}>\mathbf{2}\end{array}$ \\
\hline Symptoms & $58.8(17.2)$ & $47.6(16.1)$ & $36.4(15.3)$ & $\begin{array}{c}\mathrm{F}=50.52, \mathrm{p}<.001 \\
\mathbf{0}>\mathbf{1}>\mathbf{2}\end{array}$ \\
\hline Morning fatigue & $3.8(2.2)$ & $2.3(1.9)$ & $1.3(1.5)$ & $\begin{array}{c}\mathrm{F}=49.22, \mathrm{p}<.001 \\
\mathbf{0}>\mathbf{1}>\mathbf{2}\end{array}$ \\
\hline Evening fatigue & $5.6(2.0)$ & $4.7(2.0)$ & $3.7(2.1)$ & $\begin{array}{c}\mathrm{F}=21.41, \mathrm{p}<.001 \\
\mathbf{0}>\mathbf{1}>\mathbf{2}\end{array}$ \\
\hline Morning energy & $3.6(2.0)$ & $3.9(2.3)$ & $5.7(2.9)$ & $\begin{array}{c}\mathrm{F}=23.07, \mathrm{p}<.001 \\
\mathbf{0} \text { and } \mathbf{1}<\mathbf{2}\end{array}$ \\
\hline Evening energy & $3.3(2.0)$ & $3.6(2.0)$ & $4.7(2.1)$ & $\begin{array}{c}\mathrm{F}=14.01, \mathrm{p}<.001 \\
\mathbf{0} \text { and } \mathbf{1}<\mathbf{2}\end{array}$ \\
\hline Worst pain & $6.4(2.3)$ & $5.3(2.5)$ & $5.4(2.5)$ & $\begin{array}{c}\mathrm{F}=4.39, \mathrm{p}=.014 \\
\mathbf{0}>\mathbf{1}\end{array}$ \\
\hline
\end{tabular}

\section{CONCLUSION}

Phenotypic characteristics associated with membership in the low AFI class can be used by clinicians to identify vulnerable older patients. 